\title{
Flat Ge-doped optical fibres for food irradiation dosimetry
}

\begin{abstract}
Exposing food to radiation can improve hygiene quality, germination control, retard sprouting, and enhance physical attributes of the food product. To provide for food safety, radiation dosimetry in irradiated food is required. Herein, fabricated germanium doped (Gedoped) optical fibres have been used. The fibres have been irradiated using a gamma source irradiator, doses in the range $1 \mathrm{kGy}$ to $10 \mathrm{kGy}$ being delivered. Using Ge-doped optical fibres of variable size, type and dopant concentration, study has been made of linearity, reproducibility, and fading. The thermoluminescence (TL) yield of the fibres were obtained and compared. The fibres exhibit a linear dose response over the investigated range of doses, with mean reproducibility to within $2.69 \%$ to $8.77 \%$, exceeding the dose range of all commercial dosimeters used in evaluating high doses for the food irradiation industry. TL fading of the Ge-doped flat fibres has been found to be $<13 \%$.
\end{abstract}

Keyword: Dosimetry; Food irradiation; Ge-doped optical fibres; High dose 